\title{
论 文
}

\section{基于等温吸附实验的煤层气解吸阶段数值描述}

\author{
张政 ${ }^{\left({ }^{*}\right.}$ ，秦勇 ${ }^{\circledR}$, GuoXiong WANG ${ }^{\circledR}$ ，傅雪海 ${ }^{\circledR}$ \\ (1) 中国矿业大学资源与地球科学学院, 徐州 221116; \\ (2) School of Chemical Engineering, The University of Queensland, Brisbane, QLD 4072, Australia \\ * E-mail: zhangzheng0215@126.com
}

收稿日期: 2012-05-15; 接受日期: 2012-09-04; 网络版发表日期: 2013-07-22

国家重点基础研究发展计划(编号：2009CB219605)、国家自然科学基金重点项目(批准号：40730422)和国家重大科技专项项目(编号： 2011ZX05034-04)资助

\begin{abstract}
摘要煤层气解吸阶段的定量描述是客观认识煤层气井产能、诊断煤层气井生产状况及优化 排采管理的重要基础. 本文基于沁水盆地南部 6 口煤层气井的 12 件无烟煤样品进行了一系列的 等温吸附实验, 并以此为基础, 建立了一种以朗格缪尔吸附理论为基础的解析模型. 运用此模 型，建立了以等效解吸率及其曲线特征来刻画煤层气解吸阶段性的数值方法. 通过实验和数值 描述, 以等效解吸率曲率为依据, 提出并确定了启动压力、过渡压力和敏感压力三个定义降压 解吸阶段的关键压力点. 在此基础上, 将等温吸附实验下煤层气的解吸过程划分为四个阶段, 即零解吸阶段、缓慢解吸阶段、过渡解吸阶段和敏感解吸阶段. 通过类比分析, 发现等温吸附 实验条件下的煤层气解吸阶段性与煤层气井产气过程的异同之处, 认为较高的朗格缪尔体积和 朗格缪尔常数比有利于稳产阶段的及早到来, 但也可能使煤层气产能递减阶段提前出现.
\end{abstract}

关键词

煤层气

等温吸附

等效解吸率

关键压力

解吸阶段

数值描述
煤层气作为一种洁净高效的非常规天然气能源, 近年来受到了世界各主要产煤国家的高度重视, 而我 国作为煤炭资源大国, 煤层气资源极为丰富 ${ }^{[1]}$, 其勘 探开发对于改善我国的能源结构, 降低我国矿井瓦 斯灾害和温室效应都有着重要意义 ${ }^{[2]}$. 煤层气主要以 吸附状态赋存于煤层中, 近些年人们基于等温吸附 实验, 针对煤层甲烷的吸附性和影响因素做了大量 研究 ${ }^{[3 \sim 11]}$. 表面物理化学理论认为, 解吸是吸附的逆 过程, 属于一种传质作用 ${ }^{[12]}$. 经典的 “排水 $\rightarrow$ 降压 $\rightarrow$ 解吸 $\rightarrow$ 采气”理论 ${ }^{[13 ~ 15]}$ 推动了煤层气产业的形成与发 展, 其中降压-解吸是煤层气开发的首要环节. 煤层 气的解吸受诸多地质因素的影响, 对此前人曾做过
研究总结 ${ }^{[16]}$, 但多为对解吸率、吸附时间等解吸参数 静态的定性分析, 缺乏对解吸动态过程的定量描述, 然而解吸动态直接决定着煤层气井的产能特征. 由 等温吸附-解吸曲线可知, 煤层气的解吸是一个非线 性过程, 解吸速率和解吸量随储层压力的变化而呈 动态演变, 并呈现出一定的阶段性, 不同的阶段具有 不同的产能特性, 而这些特征都有待进一步研究.

为此, 本文基于等温吸附实验, 依据沁水盆地 6 口 煤层气井 12 件无烟煤样品的等温吸附数据进行数学变 换, 分析煤层气解吸的过程特性, 期望发展一种新的煤 层气解吸数值描述方法, 从而对煤层气排采的阶段性 进行预测, 并为煤层气井生产状况诊断提供依据.

中文引用格式: 张政, 秦勇, Wang G X, 等. 基于等温吸附实验的煤层气解吸阶段数值描述. 中国科学: 地球科学, 2013, 43: 1352-1358

英文引用格式: Zhang Z, Qin Y, Wang G X, et al. Numerical description of coalbed methane desorption stages based on isothermal adsorption experiment. Science China: Earth Sciences, 2013, 56: 1029-1036, doi: 10.1007/s11430-013-4597-2 


\section{1 样品与实验}

样品采自山西省沁水盆地南部潘庄区块 6 口煤 层气井的钻孔煤芯, 煤层埋深在 247 774 $\mathrm{m}$ 之间, 采 样层位为山西组 3 号和太原组 15 号煤层, 采样程序 按国家标准 GB/T19222-2003 执行. 同时, 收集这些 钻孔的煤层温度观测结果. 将煤样破碎到 60 80 目 $(0.25 \mathrm{~nm})$, 缩分成若干份按国家相关标准进行基本 性质测试, 1 份留待等温吸附实验. 12 件煤样的基本 性质见表 1 .

按照国家标准《煤的高压等温吸附试验方法容量
法(GB/T19560-2004)》, 利用美国 Terra Tek 公司生产 的 IS-300 型气体等温吸附/解吸仪, 在煤(储)层温度 下进行平衡水煤样的甲烷等温吸附实验,并完成了相 应的工业分析、显微煤岩组分和煤级测试工作，结果 见表 1 和图 1 .

结果显示，煤样镜质组最大反射率 $\left(R_{\mathrm{o}, \max }\right)$ 介于 $3.67 \% \sim 4.36 \%$ 之间，属于典型的无烟煤. 3 号煤层的的 朗格缪尔体积 $\left(V_{\mathrm{L}}\right)$ 为 $40.62 \sim 48.46 \mathrm{~cm}^{3} / \mathrm{g}$, 平均 45.27 $\mathrm{cm}^{3} / \mathrm{g}$; 朗格缪尔压力 $\left(P_{\mathrm{L}}\right)$ 变化在 $2.94 \sim 3.46 \mathrm{MPa}$ 之间, 平均 $3.17 \mathrm{MPa} .15$ 号煤层的朗格缪尔体积为 $34.85 \sim 46.83 \mathrm{~cm}^{3} / \mathrm{g}$, 平均 $40.55 \mathrm{~cm}^{3} / \mathrm{g}$; 朗格缪尔压力

表 1 煤样基本信息与实验测试结果 ${ }^{\text {a) }}$

\begin{tabular}{|c|c|c|c|c|c|c|c|c|c|c|c|c|}
\hline \multirow{2}{*}{$\begin{array}{l}\text { 钻孔 } \\
\text { 编号 }\end{array}$} & \multirow{2}{*}{$\begin{array}{l}\text { 煤层 } \\
\text { 编号 }\end{array}$} & \multirow{2}{*}{$\begin{array}{c}\text { 煤层温度 } \\
\left({ }^{\circ} \mathrm{C}\right)\end{array}$} & \multirow{2}{*}{$\begin{array}{c}R_{\mathrm{o}, \max } \\
(\%)\end{array}$} & \multicolumn{3}{|c|}{ 工业分析(\%) } & \multicolumn{3}{|c|}{ 显微煤岩组分 (\%) } & \multirow{2}{*}{$M_{\mathrm{e}}(\%)$} & \multicolumn{2}{|c|}{ 等温吸附 } \\
\hline & & & & $M_{\mathrm{ad}}$ & $A_{\mathrm{d}}$ & $V_{\text {daf }}$ & 镜质组 & 惰质组 & 矿物 & & $V_{\mathrm{L}}$ & $P_{\mathrm{L}}$ \\
\hline \multirow{2}{*}{ PG-01 } & 3 & 18.6 & 3.67 & 1.37 & 9.61 & 6.19 & 44.7 & 52.7 & 2.6 & 9.47 & 44.89 & 2.94 \\
\hline & 15 & 21.4 & 3.97 & 0.85 & 13.11 & 6.58 & 65.3 & 32.3 & 2.4 & 9.18 & 38.21 & 2.34 \\
\hline \multirow{2}{*}{ PG-02 } & 3 & 17 & 4.01 & 3.59 & 7.57 & 5.75 & 77.2 & 16.6 & 6.2 & 15.30 & 46.83 & 3.06 \\
\hline & 15 & 19 & 4.02 & 2.84 & 22.10 & 6.72 & 80.5 & 8.7 & 10.8 & 13.05 & 43.79 & 2.75 \\
\hline \multirow{2}{*}{ PG-03 } & 3 & 21 & 4.30 & 2.55 & 9.69 & 6.77 & 80.5 & 14.9 & 4.6 & 10.35 & 40.62 & 3.07 \\
\hline & 15 & 24 & 4.25 & 2.26 & 8.04 & 5.97 & 88.6 & 8.3 & 3.1 & 13.12 & 46.83 & 3.23 \\
\hline \multirow{2}{*}{ PG-04 } & 3 & 21 & 4.36 & 3.06 & 4.18 & 4.67 & 86.1 & 12.9 & 1.0 & 13.71 & 47.07 & 3.46 \\
\hline & 15 & 23 & 4.21 & 1.76 & 24.58 & 8.84 & 74.5 & 15.7 & 9.8 & 11.89 & 34.85 & 2.89 \\
\hline \multirow{2}{*}{ PG-05 } & 3 & 22 & 4.23 & 2.16 & 10.45 & 6.38 & 79.3 & 19.6 & 1.1 & 12.78 & 43.97 & 3.46 \\
\hline & 15 & 25 & 4.17 & 1.59 & 26.24 & 9.08 & 76.5 & 12.2 & 11.3 & 12.33 & 36.49 & 3.25 \\
\hline \multirow{2}{*}{ PG-06 } & 3 & 22.13 & 4.16 & 1.66 & 7.50 & 5.67 & 78.0 & 16.8 & 5.2 & 12.81 & 43.15 & 3.00 \\
\hline & 15 & 24.63 & 4.09 & 1.63 & 5.25 & 4.98 & 85.5 & 12.5 & 2.0 & 13.65 & 48.46 & 3.05 \\
\hline
\end{tabular}

a) $R_{\mathrm{o}, \text { max }}$, 镜质组的油浸最大反射率; $M_{\mathrm{ad}}$, 空气干燥基煤样水分含量; $A_{\mathrm{d}}$, 干燥基煤样灰分含量; $V_{\mathrm{daf}}$, 干燥无灰基煤样挥发分含量; $V_{\mathrm{L}}$, 朗格缪尔体积, $\mathrm{cm}^{3} / \mathrm{g} ; P_{\mathrm{L}}$, 朗格缪尔压力, $\mathrm{MPa} ; M_{\mathrm{e}}$, 平衡水分含量
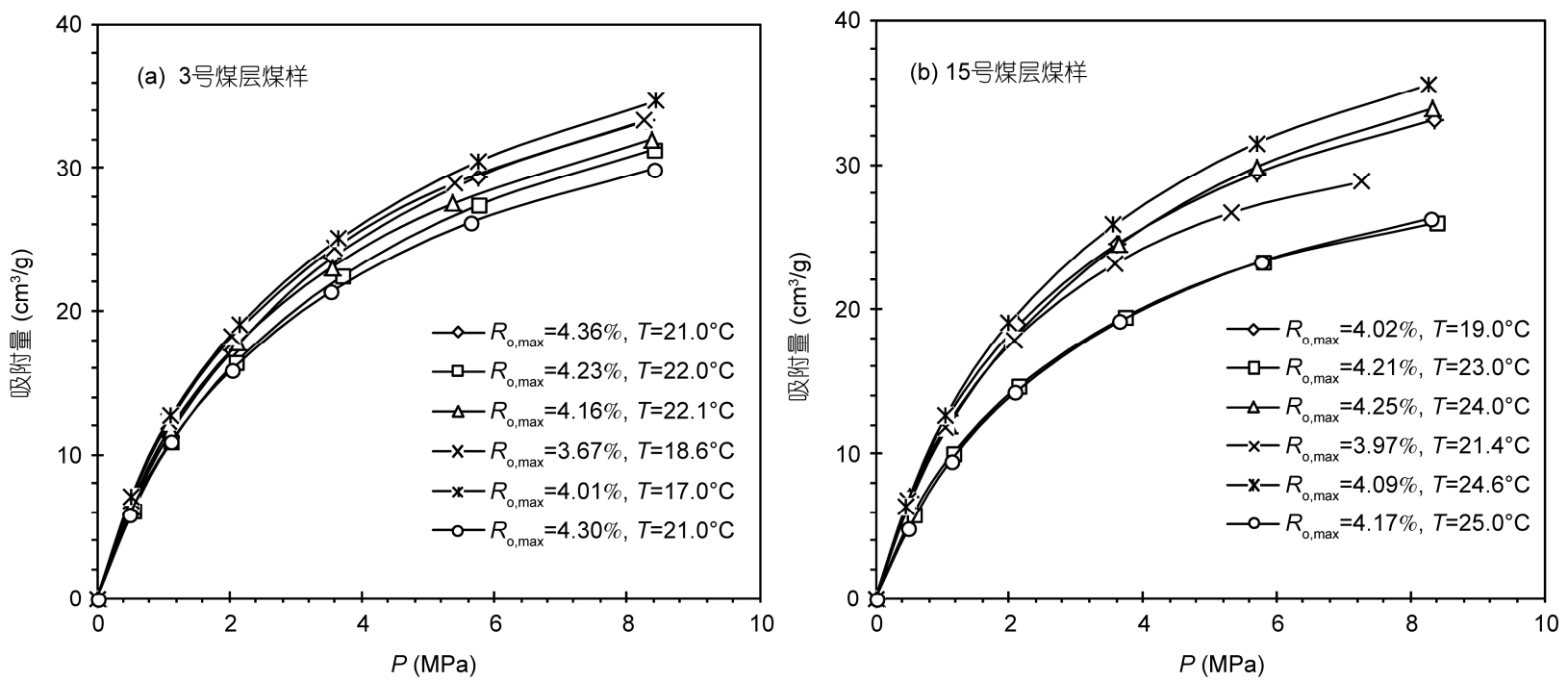

图 1 地层温度下的煤样甲烷等温吸附曲线 
介于 2.34 3.25 MPa 之间, 平均 2.91 MPa. 总体来看, 两个煤层的等温吸附常数没有实质性差别. 但是, 不 同煤样的吸附过程明显受煤级、煤岩组成和温度等因 素综合作用的影响(表 1, 图 1). 本文煤样煤级的变化 范围极小, 煤级对吸附常数的影响十分微弱(表 1). 温度对脱附起活化作用, 同一煤样温度越高, 同等压 力下的吸附量越少 ${ }^{[5,6]}$, 但温度变化对饱和吸附量的 影响不大, 由于本次研究缺少同一煤样不同温度下 的对比实验, 所以温度升高对吸附的负效应不明显. 不同显微组分吸附能力有所差异, 前人研究发现 ${ }^{[7-9]}$, 镜质组的吸附能力强于惰质组, 相同煤级富镜质组 煤的吸附能力要大于富惰质组的煤, 推其原因, 是因 为镜质组是腐植凝胶脱水及沥青化作用的产物, 变 质气孔相对发育, 导致其微孔及比表面积发育程度 高于惰质组 ${ }^{[17]}$. 由图 2 也可以看出, 研究区煤随镜质 组含量增高, 朗格缪尔体积呈增大趋势, 二者具有很 好的相关性, 朗格缪尔体积与镜惰比的关系尽管比 较离散, 但两者整体上呈现正相关趋势. 此外, 水分 含量、煤体形态等因素对吸附特性也有影响 ${ }^{[10,11]}$, 而 研究区煤的吸附特性正是众多地质因素综合作用的 结果.

\section{2 煤层气解吸阶段数值描述方法}

从表象来看, 煤层气解吸的阶段性体现为解吸 速率的显著变化. 鉴于这一原因, 可基于吸附-解吸
可逆性原理, 通过对吸附方程的数学变换来寻求描 述煤层气解吸阶段的数值方法.

储层条件下，煤对气相介质的吸附符合朗格缪 尔单分子层吸附理论 ${ }^{[18,19]}$. 本文煤样的等温吸附行 为符合朗格缪尔原理，拟合度达 $99.7 \%$ (图 1). 也就是 说，在模拟煤层温度条件下，煤的吸附量与气体压力 之间关系符合朗格缪尔方程:

$$
V=\frac{V_{\mathrm{L}} P}{P+P_{\mathrm{L}}},
$$

式中, $V$ 代表压力为 $P$ 时吸附气体的吸附量 $\left(\mathrm{cm}^{3} / \mathrm{g}\right) ; P$ 为吸附压力 $(\mathrm{MPa}) ; V_{\mathrm{L}}$ 为朗格缪尔体积 $\left(\mathrm{cm}^{3} / \mathrm{g}\right) ; P_{\mathrm{L}}$ 为 朗格缪尔压力 $(\mathrm{MPa})$.

煤层气排采是一个降压解吸过程. 为此, 本文对 理论上的解吸速率 ${ }^{[2]}$ 重新定义, 将单位压降条件下的 煤层气解吸量定义为煤层气解吸速率，称为解吸效

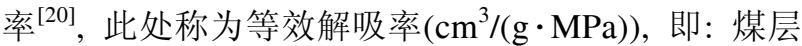
气等效解吸率 $\left(V^{\prime}=\mathrm{d} V / \mathrm{d} P\right)$ 可通过对朗格缪尔方程的一 阶求导获得:

$$
V^{\prime}=\mathrm{d} V / \mathrm{d} P=\frac{V_{\mathrm{L}} P_{\mathrm{L}}}{\left(P+P_{\mathrm{L}}\right)^{2}} .
$$

等效解吸率的变化可用等效解吸率-压力曲线的 曲率 $(K)$ 衡量:

$$
K=\frac{\left|y^{\prime \prime}\right|}{\left(1+y^{\prime 2}\right)^{3 / 2}}=\frac{\left|V^{\prime \prime \prime}\right|}{\left(1+V^{\prime \prime 2}\right)^{3 / 2}},
$$

式中, $K$ 为等效解吸率的曲率值; $y=V^{\prime}=\mathrm{d} V / \mathrm{d} P ; V^{\prime \prime \prime}=$
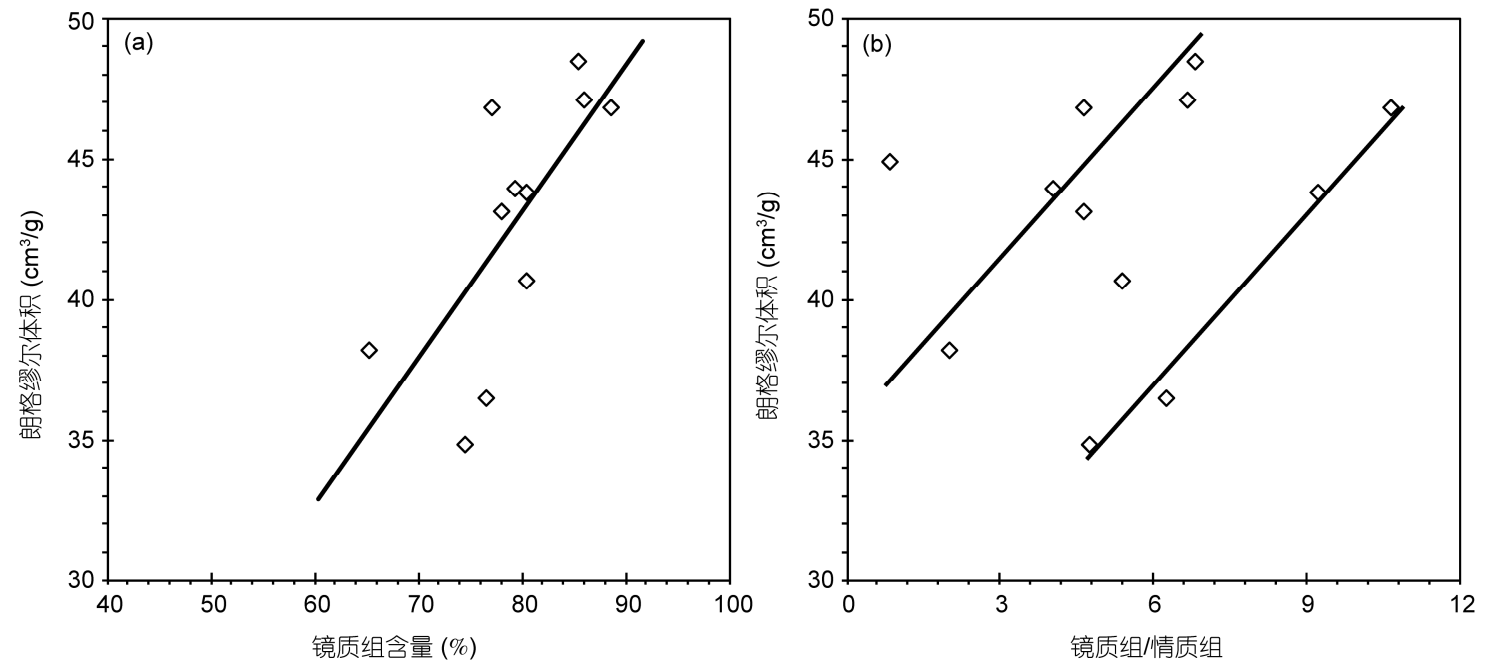

图 2 朗格缪尔体积与显微组分之间的关系 
$\mathrm{d}^{3} V / \mathrm{d} P ; V^{\prime \prime}=\mathrm{d}^{2} V / \mathrm{d} P$.

从而解得, 等效解吸率的曲率为

$$
K=\frac{\left|V^{\prime \prime \prime}\right|}{\left(1+V^{\prime \prime 2}\right)^{3 / 2}}=\frac{\frac{6 V_{\mathrm{L}} P_{\mathrm{L}}}{\left(P+P_{\mathrm{L}}\right)^{4}}}{\left\{1+\left[\frac{2 V_{\mathrm{L}} P_{\mathrm{L}}}{\left(P+P_{\mathrm{L}}\right)^{3}}\right]^{2}\right\}^{3 / 2}} .
$$

根据上述推导，等效解吸率曲线曲率变化的突 变点即为煤层气解吸阶段的分界点. 本文将类似的 突变点所对应的压力称为 “敏感压力”, 可在斜率图 上体现出来，曲率斜率用 $S$ 表示:

$$
\begin{aligned}
& S=\frac{-24 V_{\mathrm{L}} P_{\mathrm{L}}}{\left(P+P_{\mathrm{L}}\right)^{5}} \times\left\{1+\left[\frac{2 V_{\mathrm{L}} P_{\mathrm{L}}}{\left(P+P_{\mathrm{L}}\right)^{3}}\right]^{2}\right\}^{(-3 / 2)} \\
& +\frac{216\left(V_{\mathrm{L}} P_{\mathrm{L}}\right)^{3}}{\left(P+P_{\mathrm{L}}\right)^{11}} \times\left\{1+\left[\frac{2 V_{\mathrm{L}} P_{\mathrm{L}}}{\left(P+P_{\mathrm{L}}\right)^{3}}\right]^{2}\right\}^{(-5 / 2)} .
\end{aligned}
$$

下面，根据上述数学模型阐释等温吸附实验所 隐含的煤层气解吸阶段信息.

\section{3 讨论}

\section{1 解吸阶段的划分}

煤样的等效解吸率曲线如图 3. 可以看出, 等效 解吸率随压力的降低呈不断增大的趋势, 但降压初 期近似为零; 在降压的早期阶段, 随压力降低, 等效 解吸率增速缓慢; 压力进一步降低, 解吸率增幅加大, 曲线呈“下凹”型; 压力进一步降低, 解吸率迅速增大, 导致曲线斜率较大. 由此可知, 煤储层压降早期引起 的甲烷解吸量远小于后期相同压降幅度所导致的甲 烷解吸量. 换言之, 煤层气在早期解吸对压降的敏感 性较弱, 只有储层压力降低到一定程度时, 煤层气的 解吸量才能对产气量有意义，定量探讨解吸过程的 阶段性很有必要.

曲率是反映线或面弯曲程度的量化参数 ${ }^{[21]}$, 可 用来定量描述等效解吸率曲线形态的变化情况，曲 率线的变化情况又可通过其斜率体现出来. 以 PG-04 孔 15 号煤层样品为例, 反映曲率变化率的斜率曲线 存在 3 个突变点(关键压力点): $\mathrm{A}$ 为曲率变化率从零
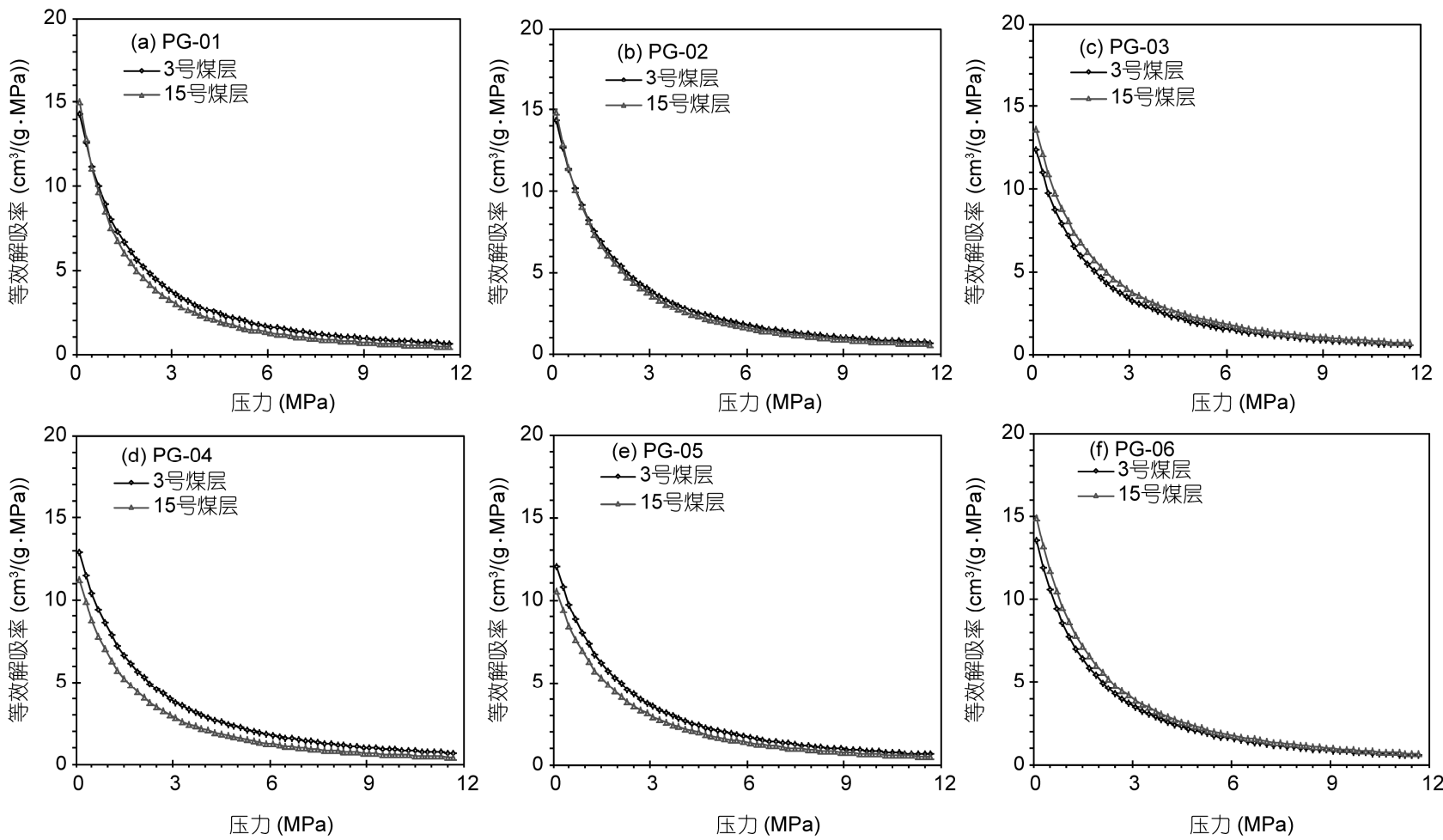

图 3 煤层气等效解吸率与压力关系曲线 
到开始增大的突变点, 该点所对应的解吸压力被定 义为启动压力; $\mathrm{B}$ 为曲率变化的负极值点, 所对应的 解吸压力称为过渡压力; $\mathrm{C}$ 为曲率变化的正极值点, 所对应的解吸压力称为敏感压力 (图 4).

根据三个关键压力点的分布特点, 可将基于等 温吸附实验的解吸过程分为四个阶段：第一阶段为 零解吸阶段, 处于降压初期, 等效解吸率极小, 相应 的其曲率及其变化值均较小, 曲率线的斜率近似为 零, 表明该阶段的压力降低基本上不导致产气; 第二 阶段为缓慢解吸阶段, 随着压力的进一步降低, 等效 解吸率曲线呈低缓的似线性增加, 曲率较小, 解吸量 仍然较低, 但增速开始变快, 表现为曲率斜率值(绝 对值)不断增大, 然后达到曲率线的负极值点; 第三 阶段为过渡解吸阶段, 压力进一步释放, 等效解吸率 曲线的斜率呈由负变正, 然后达到正极值点, 导致曲 率先增高后降低，对应的等效解吸率曲线表现为“下 凹”型, 煤层气快速解吸; 第四阶段为敏感解吸阶段, 等效解吸率曲线斜率极大, 解吸作用对压力降低最 为敏感, 单位压降所解吸的气量达到最大, 煤层气大 量解吸产出(图 5).

\section{2 与煤层气产出过程的类比分析}

煤层气井的整个排采过程一般经历排水降压( I , II, III)、稳定生产 (IV)和产量递减 (V)三大阶段 ${ }^{[22,23]}$. 生产初期需要大量的排水以降低煤储层压力, 当储 层压力降低到临界解吸压力以下时气体开始解吸产 出 ( I ); 井筒附近的储层压力首先得到释放, 致使井 筒附近的煤层气解吸, 产生第一个产气高峰( II ); 然

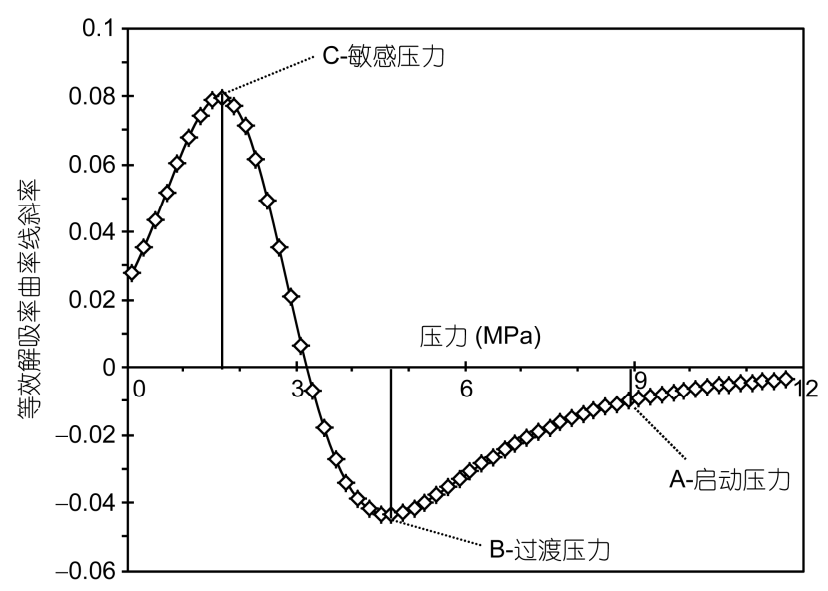

图 4 煤层气等效解吸曲率线斜率与关键压力点

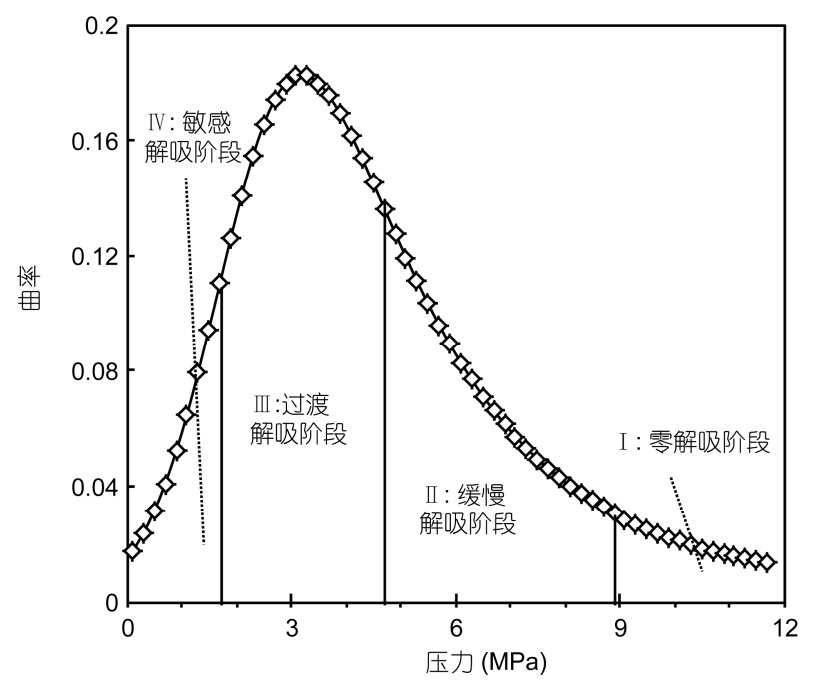

图 5 煤层气等效解吸曲率线与解吸阶段

后, 随着储层压力的进一步降低, 离井筒远端地带煤 层气受地层水饱和度及气水相对渗透率的影响, 气 源不能及时补充, 导致出现产气低谷(III). 随着排采 继续进行, 地层水饱和度不断降低, 降低到一定程度 后致使煤的水相渗透率迅速降低, 气相渗透率迅速 升高, 产气量开始回升, 达到第二个产气高峰, 产气 进入稳定阶段( IV). 稳定生产阶段过后, 大量气体已 经产出, 尽管储层压力可进一步降低, 但产气量不断 衰减( $\mathrm{V})$.

等温吸附实验下的煤层气解吸阶段与煤层气井 生产过程类似. 零解吸阶段基本不产气, 对应于 I 阶 段; 缓慢解吸阶段有少量的气体解吸, 但随着压力的 降低, 等效解吸率变化较小, 产气量增幅不大, 相当 于排采的 II 阶段; 基于等温吸附实验的煤层气解吸 不存在低谷(III)阶段, 原因在于实验样品尺寸过小, 不存在解吸气源补给的间歇期; 在过渡解吸阶段, 等 效解吸率明显增大, 解吸气量显著增加, 相当于稳产 阶段 (IV); 敏感解吸阶段单位压降煤层气的解吸量达 到最大, 而在煤层气井实际排采过程中, 产量却是递 减的 $(V)$, 这显然是相矛盾的. 分析表明有 4 方面的 原因：1）该阶段所对应的压降区间较过渡解吸阶段 明显减小；2）煤层气井排采后期, 排水作用基本停 止 $^{[22,23]}$, 储层压力降低较前期变得困难, 从而直接造 成煤层气解吸速率降低；3）随着煤层气的解吸与采 出, 高煤级煤储层渗透率显著持续减小 ${ }^{[24,25]}$, 阻碍了 煤层气的产出；4）据美国煤层气井生产经验, 煤层 气排采普遍存在枯竭压力, 约为 $0.7 \mathrm{MPa}$, 而该压降 
段以下降压困难，煤层气不能解吸.

\section{3 关键压力及其对煤层气产出过程的影响}

根据上述讨论, 过渡解吸阶段和敏感解吸阶段 对于煤层气井产量影响重大, 有必要进一步探讨过 渡压力和敏感压力的控制因素(表 2).

结果显示, 过渡压力与朗格缪尔体积呈显著的 线性正相关关系. 朗格缪尔体积增大, 达到过渡解吸 阶段的压力也在增加, 意味着缓慢解吸阶段的降压 范围缩小, 有利于稳产阶段的及早到来. 敏感压力与 朗格缪尔常数比(朗格缪尔体积与朗格缪尔压力之 比)显著线性正相关, 指示较高的朗格缪尔常数比 可能缩短稳产周期, 使得产量递减阶段提前到来(图 $6)$.

煤在平衡水条件下的煤吸附-解吸特征主要受控 于煤级, 其次为煤岩显微组分等因素 ${ }^{[17]}$. 如前所述, 本文煤样的镜质组最大反射率在 $3.67 \%$ 4.36\%之间, 变化极小, 且属于高煤化程度的无烟煤, 煤级对吸附 常数的影响十分微弱(表 1,2 ). 也正是由于高热演化
作用的结果, 不同煤岩显微组分之间的化学性质和 物理性质的差异缩小，使得显微组分组成对关键压 力以及煤层气解吸行为和解吸阶段的影响也不甚明 显.

\section{4 结论}

通过研究，本文取得如下新的认识:

(1) 建立了基于等温吸附实验的煤层气解吸阶 段解析模型和方法，提出了等效解吸率、启动压力、 过渡压力、敏感压力等煤层气解吸特征表征参数, 将 煤层气解吸过程划分为零解吸、缓慢解吸、过渡解吸 和敏感解吸四个阶段.

(2) 发现等温吸附实验条件下的煤层甲烷解吸 过程与煤层气排采产出过程之间的对应关系，认为 零解吸阶段和缓慢解吸阶段分别对应于排水降压的 第 I 阶段和第 II 阶段，过渡解吸阶段相当于稳产阶 段，敏感解吸阶段大致与产量衰减阶段对应，基于等 温吸附实验的煤层气解吸不存在煤层气井生产低谷

表 2 煤层气解吸过程中关键压力计算结果

\begin{tabular}{|c|c|c|c|c|c|c|c|c|c|}
\hline \multirow{2}{*}{ 样品编号 } & \multicolumn{2}{|c|}{ 等温吸附常数 } & \multirow{2}{*}{$\begin{array}{c}\text { 过渡压力 } \\
(\mathrm{MPa})\end{array}$} & \multirow{2}{*}{$\begin{array}{c}\text { 敏感压力 } \\
(\mathrm{MPa})\end{array}$} & \multirow{2}{*}{ 样品编号 } & \multicolumn{2}{|c|}{ 等温吸附常数 } & \multirow{2}{*}{$\begin{array}{c}\text { 过渡压力 } \\
(\mathrm{MPa})\end{array}$} & \multirow{2}{*}{$\begin{array}{c}\text { 敏感压力 } \\
(\mathrm{MPa}) \\
\end{array}$} \\
\hline & $V_{\mathrm{L}}$ & $P_{\mathrm{L}}$ & & & & $V_{\mathrm{L}}$ & $P_{\mathrm{L}}$ & & \\
\hline PG-01-3 & 44.89 & 2.94 & 5.31 & 2.05 & PG-04-3 & 47.07 & 3.46 & 5.39 & 1.89 \\
\hline PG-01-15 & 38.21 & 2.34 & 4.91 & 2.04 & PG-04-15 & 34.85 & 2.89 & 4.65 & 1.67 \\
\hline PG-02-3 & 46.83 & 3.06 & 5.42 & 2.07 & PG-05-3 & 43.97 & 3.46 & 5.19 & 1.77 \\
\hline PG-02-15 & 43.79 & 2.75 & 5.25 & 2.09 & PG-05-15 & 36.49 & 3.25 & 4.71 & 1.56 \\
\hline PG-03-3 & 40.62 & 3.07 & 5.08 & 1.82 & PG-06-3 & 43.15 & 3.00 & 5.20 & 1.95 \\
\hline PG-03-15 & 46.83 & 3.23 & 5.40 & 1.99 & PG-06-15 & 48.46 & 3.05 & 5.52 & 2.13 \\
\hline
\end{tabular}
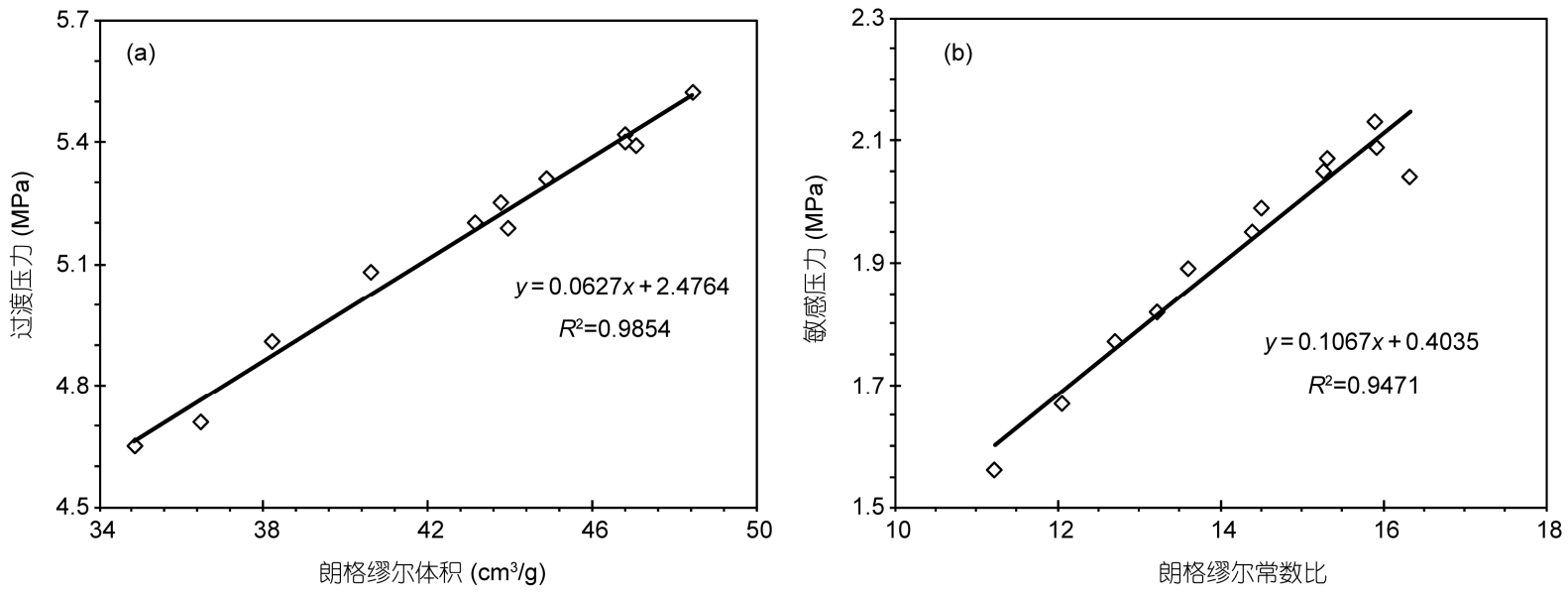

图 6 关键压力与朗格缪尔常数之间的关系 
阶段.

(3) 发现过渡压力与朗格缪尔体积以及敏感压 力与朗格缪尔常数比之间显著线性正相关, 认为较
高的朗格缪尔体积和朗格缪尔常数比一方面有利于 稳产阶段的及早到来，另一方面可能使得煤层气产 能递减阶段提前到来.

\section{参考文献}

1 秦勇. 国外煤层气成因与储层物性研究进展与分析. 地学前缘, 2005, 12: 289-297

2 傅雪海, 秦勇, 韦重蹈. 煤层气地质学. 徐州: 中国矿业大学出版社, 2007. 56-88

3 钟玲文, 张新民. 煤的吸附能力与其煤化程度和煤岩组成间的关系. 煤田地质与勘探, 1990, 4: 29-35

4 Bustin R M, Clarkson C R. Geological controls on coalbed methane reservoir capacity and gas content. Int J Coal Geol, 1998, 38: 3-26

5 张群, 崔永君, 钟玲文, 等. 煤吸附甲烷的温度-压力综合吸附模型. 煤炭学报, 2008, 33: 1272-1278

6 张庆玲, 崔永君, 曹利戈. 煤的等温吸附实验中各因素影响分析. 煤田地质与勘探, 2004, 32: 16-19

7 张群, 杨锡禄. 平衡水分条件下煤对甲烷的等温吸附特性研究. 煤炭学报, 1999, 24: 566-570

8 Crosdale P J, Beamish B B, Valix M. Coalbed methane sorption related to coal composition. Int J Coal Geol, 1998, 3: 147-158

9 钟玲文. 煤的吸附性能及影响因素. 地球科学一中国地质大学学报, 2004, 29: 327-332

10 张丽萍, 苏现波, 曾荣树. 煤体性质对煤吸附容量的控制作用探讨. 地质学报, 2006, 80: 910-915

11 降文萍, 崔永君, 钟玲文, 等. 煤中水分对煤吸附甲烷影响机理的理论研究. 天然气地球科学, 2007, 18: 576-579

12 董元彦. 物理化学. 第 3 版. 北京: 科学出版社, 2004. 222-227

13 McLennan J D, Schafer P S, Pratt T J. A Guide to Determining Coalbed Gas Content. Chicago: US Gas Research Institute, 1995

14 Saulsberry J L, Schafer P S, Schraufnagel R A. A Guide to Coalbed Methane Reservoir Engineering. Chicago: US Gas Research Institute, 1996

15 Hollub V A, Schafer P S. A Guide to Coalbed Methane Operations. Chicago: US Gas Research Institute, 1992

16 李景明, 刘飞, 王红岩, 等. 煤储集层解吸特征及其影响因素. 石油探勘与开发, 2008, 35: 52-58

17 傅雪海, 秦勇, 李贵中, 等. 特高煤级煤平衡水条件下的吸附实验. 石油实验地质, 2002, 24: 177-180

18 Gray I. Reservoir engineering in coal seams: Part I-The physical process of gas storage and movement in coal seams. SPE Reservoir Eng, 1987, 2: 28-34

19 Mavor M J, Owen L B, Pratt T J. Measurement and evaluation of coal sorption isotherm data. In: Proceedings of SPE Annual Technical Conference and Exhibition. Richardson: Society of Petroleum Engineers of AIME, 1990. 157-170

20 Qu Y J, Tang D Z, Guo B G, et al. Characteristics of coalbed methane isothermal adsorption and its controlling on productivity. In: 2011 Second International Conference on Mechanic Automation and Control Engineering. Piscataway: Institute of Electrical and Electronics Engineers, 2011. 2017-2020

21 申建, 傅雪海, 秦勇, 等. 平项山八矿煤层底板构造曲率对瓦斯的控制作用. 煤炭学报, 2010, 35: 586-589

22 杨秀春, 李明宅. 煤层气排采动态参数及其相互关系. 煤田地质与勘探, 2008, 36: 20-23

23 贺天才, 秦勇主编. 煤层气勘探开发与利用技术. 徐州: 中国矿业大学出版社, 2007.134-155

24 傅雪海, 秦勇, 张万红. 高煤级煤基质力学效应与煤储层渗透率耦合关系分析. 高校地质学报, 2003, 9: 373-377

25 陈金刚, 秦勇, 傅雪海. 高煤级煤储层渗透率在煤层气排采中的动态变化数值模拟. 中国矿业大学学报, 2006, 35: 49-52 